\title{
Prevalence of Staphylococcus aureus protein A (spa) mutants in the community and hospitals in Oxfordshire
}

\author{
Antonina A Votintseva ${ }^{1 *}$, Rowena Fung ${ }^{1,3}$, Ruth R Miller ${ }^{1,4}$, Kyle Knox ${ }^{8}$, Heather Godwin ${ }^{2,7}$, David H Wyllie ${ }^{1,5}$, \\ Rory Bowden ${ }^{6}$, Derrick W Crook ${ }^{1,2}$ and A Sarah Walker ${ }^{1,2}$
}

\begin{abstract}
Background: Staphylococcal protein A (spa) is an important virulence factor which enables Staphylococcus aureus to evade host immune responses. Genotypes known as "spa-types", based on highly variable Xr region sequences of the spa-gene, are frequently used to classify strains. A weakness of current spa-typing primers is that rearrangements in the lgG-binding region of the gene cause 1-2\% of strains to be designated as "non-typeable".

Results: We developed an improved primer which enabled sequencing of all strains, containing any type of genetic rearrangement, in a large study among community carriers and hospital inpatients in Oxfordshire, UK (6110 isolates). We identified eight novel spa-gene variants, plus one previously described. Three of these rearrangements would be designated "non-typeable" using current spa-typing methods; they occurred in 1.8\% (72/3905) asymptomatically carried and 0.6\% (14/2205) inpatient S. aureus strains. Some individuals were simultaneously colonized by both formerly non-typeable and typeable strains; previously such patients would have been identified as carrying only currently typeable strains, underestimating mixed carriage prevalence and diversity. Formerly non-typeable strains were found in more spa-types associated with multilocus sequence type ST398 (35\%), common among livestock, compared to other groups with any non-typeable strains (1-4\%), suggesting particular spa-types may have been under-represented in previous human studies.

Conclusions: This improved method allows us to spa-type previously non-typeable strains with rearrangements in the spa-gene and to resolve cases of mixed colonization with deletions in one or more strains, thus accounting for hidden diversity of $S$. aureus in both community and hospital environments.
\end{abstract}

Keywords: Staphylococcus aureus, Spa-typing, Spa-gene, Deletions, Non-typeable isolates, Community and hospital strains

\section{Background}

Staphylococcus aureus is a commensal organism that colonizes nasal mucosa in $25-30 \%$ of the healthy human population [1-6] and is responsible for a wide range of human diseases including serious nosocomial infections. $S$. aureus encodes many virulence factors including the surface Ig-binding protein A (spa) whose function is to capture IgG molecules in the inverted orientation and

\footnotetext{
* Correspondence: votintse@stats.ox.ac.uk

${ }^{1}$ Nuffield Department of Clinical Medicine, University of Oxford, John Radcliffe Hospital, Level 7, Room 7724, Oxford OX3 9DU, United Kingdom
} Full list of author information is available at the end of the article therefore prevent phagocytosis of the bacterial cells by the host immune system [7-12].

Typing the highly variable $\mathrm{Xr}$ region of the spa-gene is one of the most common methods for genotyping $S$. aureus. Even if well-established genotyping methods like MLST are indispensable, spa-typing has major advantages due to its high discriminatory power, typing accuracy, speed, reproducibility and ease of interpretation. Spa-typing also facilitates communication and data comparison between national and international clinical laboratories [13]. However, one weakness of current spa-typing methods is that rearrangements in the in the IgG-binding region of the gene, where the forward spa-primer is located, lead to 
1-2\% of strains being designated "non-typeable". Five nonspa-typeable $S$. aureus clinical strains with rearrangements in the IgG-binding domain of the spa-gene were first described by Baum et al. in 2009 [14]. Although artificially constructed spa-deficient $S$. aureus strains are used in laboratory experiments [15-18], only a few other studies have reported variants isolated from human and cattle with rearrangements in the spa-gene [19-24]. Missing particular variants that cannot be typed may affect inferences about genotype associations. Whilst the prevalence of such rearrangements can be directly estimated from the proportion of non-typeable strains, detecting rearrangements that do not affect spa-typing would require sequencing the whole spa-gene; nevertheless such rearrangements may still be informative with respect to population structure.

Further complexity is introduced by the fact that most studies type only one colony per sample, thus assuming $S$. aureus colonization is by a single strain and likely systematically underestimating the number of spa-types per individual. The presence of non-typeable $S$. aureus strains with rearrangements in the spa-gene increases the number of undetected circulating spa-types even further.

Here we therefore developed a new set of primers to amplify the spa-gene from all formerly non-typeable $S$. aureus samples regardless of the specific spa-gene rearrangement. We used our modified spa-typing protocol to investigate the nature and proportion of strains with rearrangements in the $S$. aureus spa-gene in two large studies of community nasal carriers and inpatients, and the potential impact of $S$. aureus protein A mutants on epidemiological studies.

\section{Methods}

\section{Collection of isolates}

\section{Community samples}

Nasal swab samples were collected from consenting individuals recruited from five General Practices (GP) in Oxfordshire that were members of the Thames Valley Primary Care Research Partnership, National Institute of Health Research (approved by Oxfordshire Research Ethics Committee B, reference 08/H0605/102, [25]). On recruitment, a nasal swab was taken from each individual by a research nurse. Participants were trained in selfswabbing and all participants who were culture-positive for $S$. aureus $(\mathrm{n}=360)$ on recruitment and one quarter ( $n=211)$ of initially culture-negative participants were sent a self-swabbing kit after one month and then every two months. Swabs in charcoal medium were returned by mail and stored at $4^{\circ} \mathrm{C}$ before processing. During the 36 months of the study, S. aureus was isolated from at least one swab of 442 individuals yielding 3905 samples which were spa-typed and analyzed here.

\section{Inpatient samples}

$S$. aureus isolates were obtained from samples collected from the Intensive care Unit (ITU), Gerontology and Trauma wards of the John Radcliffe hospital in Oxford as a part of routine screening for inpatients for infection control surveillance. For all three wards, nasal swabs were collected from individuals at ward admission and discharge as well as once a week during their stay within the ward [26]. All swabs were taken by nurses, as described above, and were processed by the routine laboratory at the John Radcliffe hospital, Oxford. In total, S. aureus was isolated from 2205 samples from 1273 inpatients (ITU: 1338 samples, 784 individuals; Gerontology: 134 samples, 72 individuals; Trauma: 733 samples, 417 individuals) which were spa-typed and analysed here.

\section{Isolation of S. aureus and DNA extraction}

Each nasal swab was placed in $5 \% \mathrm{NaCl}$ enrichment broth (E and $\mathrm{O}$ Laboratories) and incubated overnight at $37^{\circ} \mathrm{C}$. A loopful of enrichment broth was sub-cultured onto SaSelect chromogenic agar (Bio-Rad) and incubated at $37^{\circ} \mathrm{C}$ overnight. Pink/orange colonies regarded as $S$. aureus were positively identified using a Prolex $^{\mathrm{Tm}}$ Staph Xtra Latex Kit (Pro-Lab Diagnostics) and catalase, DNAse and tube coagulase tests. Methicillin resistance was tested on columbia agar with $5.0 \%$ salt (Oxoid) with $\mathrm{BBL}^{\mathrm{m}}$ Sensi-Disc $^{\mathrm{Tm}} 1 \mu \mathrm{g}$ Oxacillin discs (BD).

Mixed glycerol stocks of $S$. aureus cultures were prepared by suspending several loopfuls of bacteria taken by sweeping across the SaSelect plate in $1.5 \mathrm{ml}$ of saline (E and O Laboratories) with $200 \mu \mathrm{l}$ of $45 \%$ glycerol for storage at $-80^{\circ} \mathrm{C}$. Taking a sweep across the plate rather than picking a single colony for glycerol stocks allowed us to maintain the genetic diversity of nasal strains in the sample for later analyses.

Crude S. aureus DNA extracts ('boilates') used for spatyping were made from mixed glycerol stocks revived on SaSelect plates. Using a $1 \mathrm{~mm}$ loop, a small amount of bacteria was emulsified into $60 \mu \mathrm{l}$ of Tris-EDTA (TE) buffer (Sigma-Aldrich), then heated in a thermocycler at $99.9^{\circ} \mathrm{C}$ for 10 minutes and centrifuged at $13,200 \times g$ for 2 minutes. $40 \mu \mathrm{l}$ of supernatant was removed without disturbing the pellet and stored at $-20^{\circ} \mathrm{C}$ for use as a PCR template.

\section{Spa-typing}

A staged spa-typing protocol was developed to enable identification of multiple-strain colonization on a largescale [27]. The polymorphic $\mathrm{X}$ region of the protein $\mathrm{A}$ gene (spa) was amplified with primers $1095 \mathrm{~F}$ : 5' -AGAC GATCCTTCGGTGAGC-3' and 1517R: 5'-GCTTTTGC AATGTCATTTACTG-3' [28,29]. PCR reactions consisted of $0.25 \mathrm{mM}$ dNTPs (Qiagen), 0.5 U of GoTaq Flexi DNA Polymerase (Promega), Colorless GoTaq Flexi 
Buffer, $2.5 \mathrm{mM}$ of magnesium chloride and $0.25 \mu \mathrm{M}$ of primers in a volume of $10 \mu \mathrm{l}$. PCR conditions were $94^{\circ} \mathrm{C}$ for $2 \mathrm{~min}$; 35 cycles each of $94^{\circ} \mathrm{C}$ for $30 \mathrm{~s}, 50^{\circ} \mathrm{C}$ for $30 \mathrm{~s}$, and $72^{\circ} \mathrm{C}$ for $60 \mathrm{~s}$; and a final extension at $72^{\circ} \mathrm{C}$ for 5 min. PCR products were purified using Agencourt AMPure XP beads (Beckman Coulter).

Samples were sequenced with the same primers as used in PCR. Sequencing reactions used BigDye v3.1 sequencing mix (Applied Biosystems) and were cycled using 30 cycles of $96^{\circ} \mathrm{C}$ for $10 \mathrm{~s}, 50^{\circ} \mathrm{C}$ for $5 \mathrm{~s}$, and $60^{\circ} \mathrm{C}$ for 2 min. Products were purified with Agencourt CleanSEQ beads (Beckman Coulter) and separated on an ABI 3730 DNA Analyzer (Applied Biosystems).

Chromatograms were analyzed using Ridom StaphType v2.0.3 software (Ridom $\mathrm{GmbH}$ ). The relationships between spa-types were investigated using the BURP clustering algorithm [30] incorporated into Ridom StaphType.

\section{Identification of rearrangements in spa-gene}

A small proportion of isolates did not yield clean sequence traces with the original primers indicating the presence of rearrangements in the spa-gene. To identify possible rearrangements, primers spa-3 F: 5'-ATAGCGTGATTTTG CGGTT-3' and spa-3R: 5' -CTAAATATAAATAATGTT GTCACTTGGA-3' [14] were used to amplify the whole spa-gene. As some isolates failed to amplify even with this extended set of primers, an alternate forward primer, spaT3-F: 5'-CAACGCAATGGTTTCATCCA-3' binding upstream from $1095 \mathrm{~F}$ was used together with standard reverse primer 1517R. Primer spaT3-F binds to a part of sequence encoding an IgG-binding domain of the spagene that is repeated five times in the gene (Figure 1). Due to presence of multiple binding sites for the spaT3-F primer within spa-gene, only the reverse primer (1517R) was used for sequencing.

To identify rearrangements in the spa-gene which do not affect standard spa-typing, a subset of 32 community samples and 67 bacteraemia samples were sequenced with both spa-3 F/spa-3R and spaT3-F/1517R sets of primers. 67 bacteraemia samples were randomly selected from previously existed collection of hospital invasive isolates.

Sequences were analysed using ProSeq v3.2 (http:// dps.plants.ox.ac.uk/sequencing/proseq.htm).

Example sequences for each type of the spa-gene variant have been deposited in the GenBank under the accession numbers JX912490 to JX912498.

\section{Statistical analyses}

Fisher's exact test, Chi square test and 5x2 exact test were used to compare categorical variables between groups. $P$ values $<0.05$ were considered statistically significant.

\section{Results and discussion}

Identification of rearrangements in the spa-gene

Within two large longitudinal studies of $S$. aureus carriage in the community (3905 isolates) [25] and hospital (2205 isolates) [26] several non-typeable $S$. aureus strains were identified using standard spa-primers (1095 F/1517R) [14]. Isolates from both studies were spa-typed using a staged protocol, developed to resolve single- and multiplestrain colonization [27]. According to the protocol, spasequences were classified as follows: (i) clean sequence traces were interpreted as single strain colonisation, (ii) mixed sequence traces, characterised by distinct double peaks, were interpreted as putative multiple strain colonization, and (iii) unreadable sequence traces represented failed samples, which were retyped. Samples with mixed sequence traces were further resolved by isolating 12 individual colonies; if typing of individual colonies failed, strains were considered non-typeable with standard primers.

Sequence traces of non-typeable samples showed either complete lack of amplification, or mixed sequence traces from both DNA boilates of mixed glycerol stock and of 12 individual colonies. As previously shown [14], nontypeability of $S$. aureus strains can be attributed to

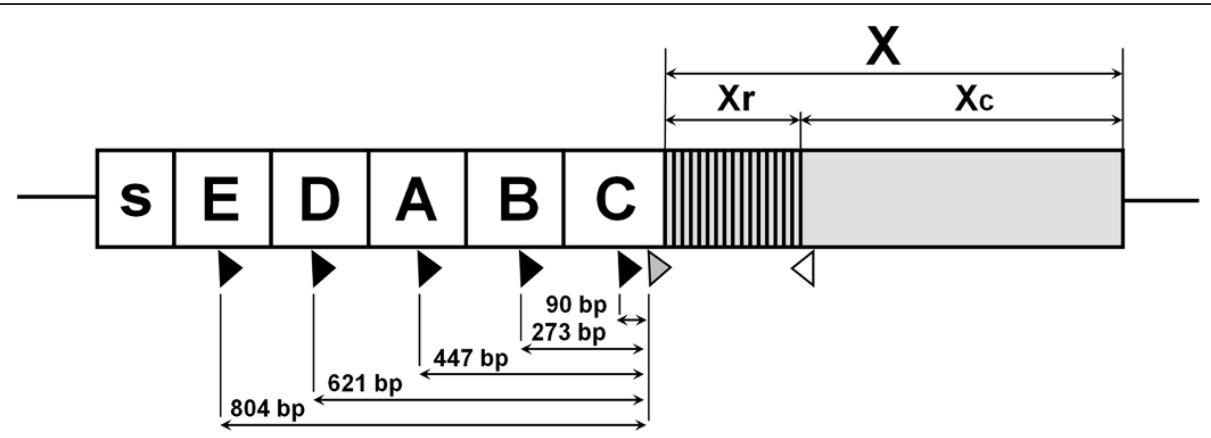

Figure 1 Scheme of the spa-gene with annealing sites for the novel spaT3-F primer and standard primers. Notes: black arrows indicate five annealing sites for spaT3-F primer; grey arrow indicates annealing site for $1095 \mathrm{~F}$ standard primer; white arrow indicates annealing site for $1517 \mathrm{R}$ standard primer; figures represent distance between the beginning of spaT3-F primer and the beginning of Xr region. Spa-gene: $\mathrm{S}-$ signal sequence, E, $D, A, B, C$ - lgG-binding domains, $X$ - region which lacks lgG-binding activity and consists of repetitive region ( $\mathrm{Xr}$ ) and C-terminal region (Xc). 
deletions in the spa-gene, explaining the lack of amplification in some of our samples. However the persistence of mixed sequence traces that could not be resolved by typing individual colonies indicated the presence of other types of spa-gene rearrangements.

To identify the nature of rearrangements in all our non-typeable strains we designed a new forward spaT3-F primer and combined it with reverse primer $1517 \mathrm{R}$, used for routine spa-typing [29]. Primer spaT3-F has a binding site in each of the five IgG-binding domains of the spa-gene upstream of the repetitive $\mathrm{Xr}$ region (Figure 1) and resulted in up to five staged PCR products per sample, depending on the type of rearrangements in the IgGbinding region (Figure 2). Due to its multisite binding within the spa-gene, the spaT3-F primer could be used to type samples with deletions of up to four IgG-domains of the spa-gene and to detect and type samples with mixtures of $S$. aureus strains with and without deletions.

With the novel spaT3-F/1517R primer set we were able to type $100 \%$ of samples that could not be spatyped using the standard current set of primers (denoted "formerly non-typeable").
In total, we found eight completely novel deletions/insertions in the IgG-binding region of the spa-gene plus one deletion that has been reported before [14], in 6110 community and inpatient $S$. aureus strains from Oxfordshire (Figure 3). We never observed the deletion of the whole or a part of the repetitive $\mathrm{Xr}$ region in $S$. aureus, in contrast to Baum at al who described partial or total deletions of the $\mathrm{Xr}$ region in three bacteraemia isolates [14]: our large study suggests this happens extremely rarely in carriage. One explanation for the difference may be that Baum et al. considered disease-causing isolates while most of our community and hospital isolates were carriage.

As expected, non-typeable samples that did not amplify with the standard set of primers had one of two types of deletions affecting the binding site for the original forward primer. Deletion E (174 bp) was previously described by Baum at al. in a clinical S. aureus strain [14]. Deletion G (63 bp) is a novel deletion always paired with insertion B (63 bp) (Figure 3). Non-typeable samples with persistent mixed sequence traces revealed the presence of the insertion C2 (174 bp) (Figure 3). This insertion contains
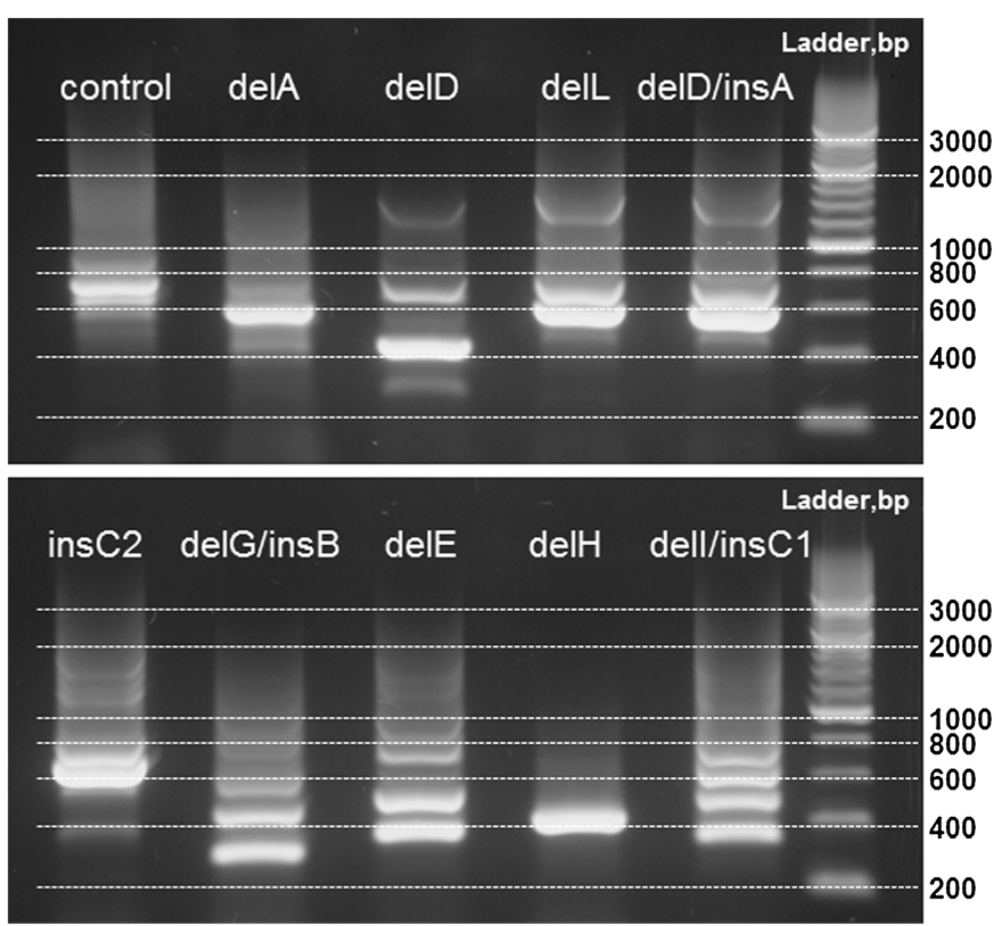

Figure 2 Amplification of spa-locus with novel primers spaT3-F/1517R from the samples with rearrangements in the spa-gene. A control sample does not have any rearrangements in the spa-gene and shows five PCR bands according to five annealing sites of the spaT3-F primer, including a faint band in the region of $500 \mathrm{bp}$. DelH shows one thick band most likely consisted of two merged bands from the two spaT3-F annealing sites that had been brought close together by the deletion. InsC2 has a bright band (600 bp) most likely consisted of two PCR products due to insertion of additional spaT3-F annealing site. The rest of the samples display the number of bands according to the types of rearrangements (Figure 3). Amplification of these samples with the standard spa-typing primers $1095 \mathrm{~F} / 1517 \mathrm{R}$ will give no bands for the samples with delE and delG, which affect the position of $1095 \mathrm{~F}$ standard primer. For the rest of the sample PCR will generate a single band (double band for the insC2) located at a variable position on the ladder depending on the number of repeats within Xr region of each sample. 


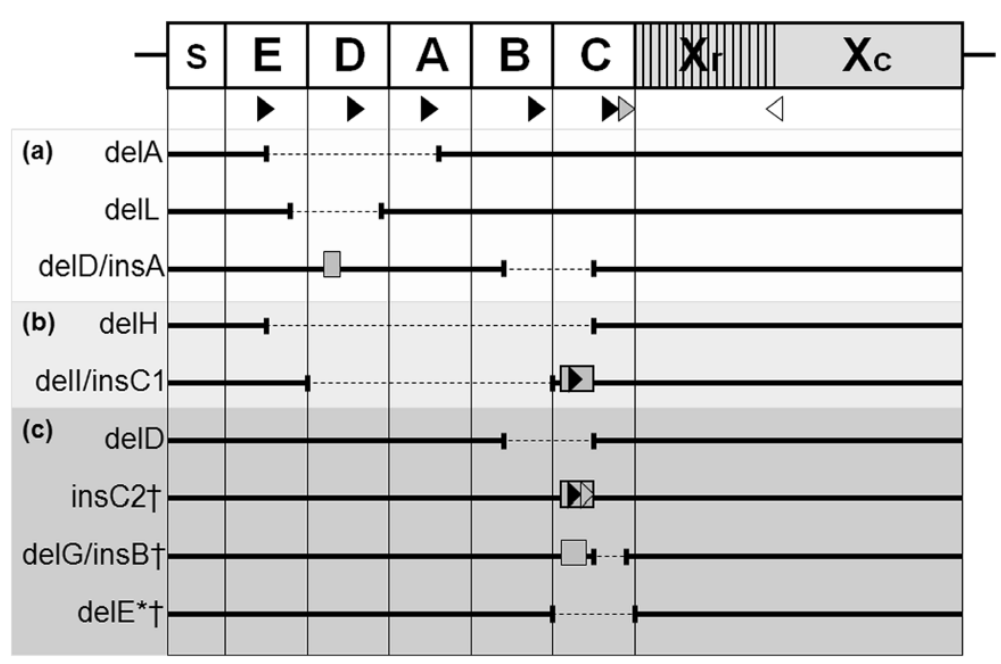

Figure 3 Scheme of the rearrangements identified in the IgG-binding domains of spa-gene in samples from Oxfordshire. Notes: The insertions are indicated by grey rectangles. The deletions indicated by dotted thin lines. Black arrows indicate annealing sites for spaT3-F novel primer; grey arrows indicates annealing site for $1095 \mathrm{~F}$ standard primer; white arrow indicates annealing site for 1517R standard primer. Grey rectangles with arrows indicate insertions with additional binding sites for primers. Panel (a) indicates deletions found only in community samples, panel (b) indicates deletions found only in inpatient samples and panel (c) indicates deletions found both in community and inpatient samples. Spa gene: $\mathrm{S}$ - signal sequence, E, D, A, B, C sequences encoding IgG-binding domains, $\mathrm{X}$ - region which lacks lgG-binding activity and consists of repetitive region $\left(X_{r}\right)$ and C-terminal region $\left(X_{C}\right)$. Asterisk indicates deletion previously described by Baum et al., 2009. Dagger indicates deletions/insertions leading to strains being designated non-typeable using the standard primers.

additional binding sites for the spaT3-F and original spa-forward primer, producing two PCR products and distinct double peaks in sequence traces when sequenced with the original spa-forward primer. Sequencing from the reverse primer (1517R) produced clean sequence traces without double peaks.

Surprisingly, in some samples that did not amplify with the standard primer set we found rearrangements represented by deletion A (357 bp) and deletion D/insertion A (174 bp/10 bp) that do not affect the position of the standard forward primer. To investigate the presence of deletions that do not affect spa-typing and therefore can remain unnoticed, we sequenced the whole spagene from 32 community carriage and 67 bacteraemia isolates chosen at random from the previously spa-typed collection. We found four novel deletions, deletion D (174 bp) in both bacteraemia and community strains, deletion L (183 bp) only in community strains, deletion $\mathrm{H}$ (705 bp) and deletion I/insertion C1 (531 bp/ 174 bp) only in bacteraemia isolates (Figure 3 ). The largest deletions of three to four IgG-binding domains were found only in S. aureus bacteraemia strains.

Therefore, the presence of different types of deletions and insertions in the spa-gene, identified by spaT3-F/ 1517R primers, demonstrates that $S$. aureus colonization/ infection is highly complex. People may have a single strain without rearrangements, with deletions that do not affect spa-typing, or with rearrangements that do affect spa-typing. Alternatively, they may carry multiple strains without deletions in any strain, with 'hidden' deletions that do not affect spa-typing in one or more strains, or with rearrangements that do affect spa-typing in one or more strains.

\section{Prevalence of spa-gene rearrangements in community and hospital strains}

Spa-typing of 3905 community S. aureus isolates and 2205 hospital isolates using the staged spa-typing protocol showed that $1.8 \%(\mathrm{n}=72)$ of samples from $1.8 \%$ community carriers and $0.6 \%(\mathrm{n}=14)$ of samples from $0.7 \%$ inpatients were formerly non-typeable (Table 1 ). Significantly more strains from individuals in the community were formerly non-typeable compared with hospital inpatients ( $\mathrm{p}<0.0001$ ), and there was also a trend towards more individuals carrying formerly non-typeable strains in the community than hospital $(\mathrm{p}=0.053)$. One explanation may be because more longitudinal samples were collected per individual for the community compared with the inpatient study (maximum 20 versus 4 samples respectively), providing more opportunity to encounter non-typeable strains in individuals in the community study.

The proportion of $S$. aureus strains with 'hidden' deletions in the IgG-binging region of the spa-gene that do not affect spa-typing was estimated using spaT3-F/ $1517 \mathrm{R}$ primers on a random subset of previously typed samples. These hidden spa-gene deletions were found in $11 \%(6-19 \%)$ of S. aureus strains from $11 \%(6-19 \%)$ of individuals (Table 2). 
Table 1 S. aureus isolates with and without different types of rearrangement in the spa-gene in community and inpatient samples: formerly non-typeable isolates

\begin{tabular}{|c|c|c|c|c|c|c|c|c|}
\hline \multirow[t]{3}{*}{ Group } & \multicolumn{4}{|c|}{ Community $^{1}$} & \multicolumn{4}{|c|}{ Hospital $^{2}$} \\
\hline & \multicolumn{2}{|r|}{ Isolates } & \multicolumn{2}{|r|}{ Individuals } & \multicolumn{2}{|r|}{ Isolates } & \multicolumn{2}{|r|}{ Individuals } \\
\hline & no. & $\%$ & no. & $\%$ & no. & $\%$ & no. & $\%$ \\
\hline Total & 3,905 & $100 \%$ & 442 & $100 \%$ & 2,205 & $100 \%$ & 1,273 & $100 \%$ \\
\hline $\begin{array}{l}\text { Pure without deletions/insertions } \\
\text { or with hidden deletions }{ }^{3}\end{array}$ & 3647 & $93.4 \%$ & 334 & $75.6 \%$ & 2055 & $93.2 \%$ & 1150 & $90.3 \%$ \\
\hline $\begin{array}{l}\text { Mixed with or without deletions } \\
\text { and/or rearrangements }{ }^{4}\end{array}$ & 258 & $6.6 \%$ & 108 & $24.4 \%$ & 150 & $6.8 \%$ & 123 & $9.7 \%$ \\
\hline \multirow{2}{*}{$\begin{array}{l}\text { Formerly non-typeable: i.e. pure } \\
\text { with rearrangements affecting } \\
\text { standard spa-typing }\end{array}$} & \multirow[t]{2}{*}{72} & $1.8 \%$ (from total) & \multirow[t]{2}{*}{8} & $1.8 \%$ (from total) & \multirow[t]{2}{*}{14} & 0.6\% (from total) & \multirow[t]{2}{*}{9} & 0.7\% (from total) \\
\hline & & 27.9\% (from 12 picks) & & 7.4\% (from12 picks) & & 9.3\% (from 12 picks) & & 7.3\% (from 12 picks) \\
\hline
\end{tabular}

Thus up to $13 \%$ of $S$. aureus carriers could, at some point, be colonized with a strain that has deletions/ insertions in the IgG-binding region of the spa-gene, $2 \%$ carrying completely 'non-typeable' strains.

Spa-gene rearrangements lead mixed S. aureus colonization in humans to be underestimated

The staged spa-typing protocol allowed us to detect the simultaneous presence of two or more strains in $11 \%$ of $S$. aureus carriers. However, the presence of deletions that affect spa-typing in one or more strains within the mixture complicates the typing process and leads to underestimation of the prevalence of multiple colonization and number of strains involved.

The problem is illustrated using nine samples obtained over 14 months from one community nasal carrier $\mathrm{AE}$ (Table 3). Spa-typing with the standard primer sets (1095 F/1517R) demonstrated that AE only ever carried one strain, which was type $\mathrm{t} 230$ at most time points, the spa-type t008 on one occasion and one non-typeable strain. Re-typing the same DNA extractions with our alternative novel primers (spaT3-F/1517R) showed that all samples had mixed sequence traces, apart from the formerly non-typeable strain that had deletion $\mathrm{E}$

Table 2 S. aureus isolates with and without different types of rearrangement in the spa-gene in community and inpatient samples: isolates with hidden deletions

\begin{tabular}{|c|c|c|c|c|}
\hline \multirow[t]{2}{*}{ Group } & \multicolumn{2}{|c|}{ Isolates } & \multicolumn{2}{|c|}{ Individuals } \\
\hline & no. & $\%$ & no. & $\%$ \\
\hline $\begin{array}{l}\text { Total strains without deletions/insertions or } \\
\text { with only hidden deletions investigated }\end{array}$ & 99 & $100 \%$ & 97 & $100 \%$ \\
\hline Hidden deletions found & 11 & $11 \%$ & 11 & $11 \%$ \\
\hline
\end{tabular}

Note: Hidden deletions were found in $16 \%$ (5/32) of S. aureus strains from $16 \%$ $(5 / 31)$ individuals in the community and in $9 \%(6 / 67)$ strains from $9 \%(6 / 66)$ hospital in patients with bacteraemia $(p=0.33)$; pooled data are therefore presented. associated with spa-type t012. Therefore, 12 single colonies were isolated for each sample and re-typed with alternative primers. This identified five spa-types carried by $\mathrm{AE}$ at various time points, and mixed strain colonization by two-three spa-types on four occasions, including two strains with deletion E. We were unable to resolve all samples by typing 12 individual colonies, even though they showed presence of mixed sequence traces (time points 4,10,12 and 14), which could be explained by a low frequency of one of the colonizing strains.

The limitations of the conventional spa-typing protocol make it impossible to identify and type $S$. aureus strains with rearrangements in the spa-gene in individuals with

Table 3 Spa-typing of S. aureus strains from a single individual AE with two sets of primers: standard primers 1095 F/1517R and novel primers spaT3-F/1517R

\begin{tabular}{|c|c|c|c|}
\hline \multirow{2}{*}{$\begin{array}{l}\text { Time } \\
\text { points, } \\
\text { months }\end{array}$} & \multicolumn{2}{|c|}{ DNA prep (mixed boilate) } & \multirow{2}{*}{$\begin{array}{c}12 \text { single colony picks }{ }^{2} \\
\text { spaT3-F/1517R }\end{array}$} \\
\hline & $1095 \mathrm{~F} / 1517 \mathrm{R}$ & spaT3-F/1517R & \\
\hline$\overline{A E-0}$ & $\mathrm{t} 230$ & $\mathrm{MST}^{1}$ & $\mathrm{t} 230 / \mathbf{t 0 1 2}$ \\
\hline$A E-1$ & non-typable & to12 & t012 \\
\hline$A E-2$ & $\mathrm{t} 230$ & MST & $\mathrm{t} 230 / \mathbf{t} 012$ \\
\hline$A E-4$ & $\mathrm{t} 230$ & MST & $\mathrm{t} 230$ \\
\hline$A E-6$ & $\mathrm{t} 230$ & MST & t230/t528 \\
\hline $\mathrm{AE}-8$ & t008 & MST & t008/t012/t571 \\
\hline$A E-10$ & $\mathrm{t} 230$ & MST & $\mathrm{t} 230$ \\
\hline$A E-12$ & $\mathrm{t} 230$ & MST & $\mathrm{t} 230$ \\
\hline$A E-14$ & $\mathrm{t} 230$ & MST & t230 \\
\hline
\end{tabular}

${ }^{1}$ mixed sequence traces; ${ }^{2}$ spa-types in bold have delE and could not be typed with standard primers; spa-repeats:

t230: 08-16-02-16-34.

t571: 08-16-02-25-02-25-34-25.

t008: 11-19-12-21-17-34-24-34-22-25.

t012: 15-12-16-02-16-02-25-17-24-24.

t528: 04 . 
multiple strain colonization. The staged spa-typing protocol allows us to resolve cases of mixed strain colonization with deletions in one or more strains. Even 12 single colony picks could not always identify the presence of low-frequency strains with deletions, illustrating the even greater challenge of estimating the proportion of non-typeable strains within mixed colonization. Thus diversity in colonizing and infecting strains is inevitably underestimated.

\section{Inpatients' strains can acquire deletions in the spa-gene}

We also found that $S$. aureus strains can acquire deletions in the spa-gene during inpatients' hospital admission. Such acquisition of deletions was never observed for longitudinal carriage strains from individuals in the community, with those deletions observed being present from the first time the strain carrying the deletion was identified (Additional file 1: Table S1).

Among six hospital patients with deletions that affect spa-typing, three individuals (BA, BB and $\mathrm{BF}$ ) already carried the strain with the deletion when they were admitted. The three other patients (BC, BD and $\mathrm{BE}$ ) acquired a deletion during their hospital stay on the background of a spa-type carried at admission (Table 4). There were eight, four and six days between the last swab without and the first swab with the acquired deletion for $\mathrm{BC}, \mathrm{BD}$ and $\mathrm{BE}$ respectively. All three patients acquiring deletions during hospital admission either had long-term illnesses and/or had taken several antibiotics (BC: teicoplanin; BD: doxycycline; BD: flucloxacillin, penicillin, ciprofloxacin, vancomycin, erythromycin, gentamicin, tetracycline).

The repetitive nature of the spa-gene makes it unstable and highly prone to internal rearrangements, which in

Table 4 Individuals who acquired a deletion in the $S$. aureus spa-gene during their hospital admission

\begin{tabular}{|c|c|c|c|c|}
\hline Individual ID & $\begin{array}{c}\text { Date swab } \\
\text { taken }\end{array}$ & Results & Spa type & Rearrangements \\
\hline$B C$ & $30 / 01 / 2011$ & MSSA & t298 & \\
\hline$B C$ & 08/02/2011 & MSSA & t298 & delG-insB \\
\hline$B D$ & $14 / 04 / 2011$ & MSSA & $\mathrm{t} 571$ & \\
\hline $\mathrm{BD}$ & 19/04/2011 & MSSA & t571 & delG-insB \\
\hline $\mathrm{BD}$ & $26 / 04 / 2011$ & MSSA & t571 & delG-insB \\
\hline$B E-a^{1}$ & $20 / 06 / 2011$ & MSSA & $\mathrm{t} 179$ & \\
\hline$B E-g^{2}$ & 20/06/2011 & MSSA & t179 & \\
\hline$B E-n^{3}$ & $20 / 06 / 2011$ & MSSA & t179/t078 & \\
\hline BE-th ${ }^{4}$ & 20/06/2011 & MSSA & t179/t078 & \\
\hline $\mathrm{BE}$ & 05/07/2011 & MSSA & t179/t078 & \\
\hline $\mathrm{BE}$ & $12 / 07 / 2011$ & MSSA & t179/t078 & delE \\
\hline $\mathrm{BE}$ & 20/07/2011 & MSSA & $\mathrm{t} 179 / \mathbf{t 0 7 8}$ & delE \\
\hline
\end{tabular}

${ }^{1-4}$ body sites swabs: a - axilla, $\mathrm{g}$ - groin, $\mathrm{n}$ - nose, th - throat; all other swabs are nasal swabs; spa-types in bold acquired deletion that affects binding site for standard forward spa-typing primer. bacteria occur via either RecA-dependent or RecAindependent recombination [31-33]. These rearrangements might have positive or negative effects as protein $\mathrm{A}$ is an important virulence factor that plays a central role in $S$. aureus defence against the host immune response. There is new evidence that the antibiotic ciprofloxacin increases the intrachromosomal DNA recombination rate in Escherichia coli [34]. Other antibiotics might potentially have similar effects, yet undiscovered. Taking into account that the three inpatients who acquired deletions during their stay at the hospital had been taking specific antibiotics for a long time or a wide range of antibiotics for a short period, including ciprofloxacin, it is possible that antibiotic pressure might be one factor that drives genetic rearrangements in the $S$. aureus protein A gene. However, we also cannot exclude the possibility that these deletions may have been present already at low frequency, and undetected, before increasing to become the majority variant (rather than being acquired de novo). Nevertheless this scenario also would support antibiotics playing a role in emergence of deletions to detectable levels.

In the community, most individuals colonized by $S$. aureus strains carry them without displaying any symptoms. However, when some of them became invasive, the change of habitat, for example on a background of antibiotic pressure, might promote acquisition of rearrangements in the spa-gene that might be advantageous in new environment even if they lead to loss or change of protein function. As shown previously, progress of infectious disease is typically associated with acquisition of mutations or rearrangements in the pathogen, many of which lead to loss of function in a number of genes [35].

\section{Deletions appear to be over-represented in clonal line- ages related to livestock}

In total, we found 20 spa-types from 33 individuals associated with nine types of rearrangements in the spa-gene (Additional file 2: Table S2). All types of deletions were associated with a mixture of related and unrelated spatypes, only insertion $\mathrm{C} 2$ was associated with a group of 3 closely related spa-types: t021, t012 and t10173. The 20 spa-types with rearrangements were clustered into five groups of closely-related variants and five non-related singletons (Table 5).

Any clusters of related spa-types with a higher prevalence of rearrangements affecting spa-typing (delE, delG-insB or insC2) would be likely to be underrepresented, or even missing, in the majority of studies based on routine spa-typing protocols. To test this hypothesis, we compared the proportion of individuals with and without rearrangements affecting spa-typing in the four groups of spa-types and the singleton that we found had one or more rearrangements affecting spa-typing $(5 \times 2$ exact test, Table 5). In group $135 \%$ of strains were 
Table 5 Spa-types and groups in which deletions/insertions in the spa-gene were observed

\begin{tabular}{|c|c|c|c|c|c|}
\hline Spa-types & Spa-repeats & $\begin{array}{l}\text { Individuals with } \\
\text { deletions, } \\
\text { no. (column \%) }\end{array}$ & $\begin{array}{c}\text { Hidden } \\
\text { deletions } \\
\text { not affecting } \\
\text { spa-typing } \\
\text { (no.) }\end{array}$ & $\begin{array}{l}\text { Deletions/ } \\
\text { insertions } \\
\text { affecting } \\
\text { spa-typing } \\
\text { (no.) }\end{array}$ & $\begin{array}{l}\text { Individuals } \\
\text { with deletions } \\
\text { affecting spa-typing/ } \\
\text { total individuals with } \\
\text { this spa-type }\end{array}$ \\
\hline Group 1 & & $7(21 \%)$ & & & $7 / 20(35 \%)^{*}$ \\
\hline t571 & $08-16-02-25-02-25--------34-25$ & $6(18 \%)$ & & $\begin{array}{l}\text { delG-insB(5); } \\
\text { delE(1) }\end{array}$ & $6 / 19(32 \%)$ \\
\hline t3085 & $08-16-02-25-02-25-34-25-34-25$ & $1(3 \%)$ & & delE (1) & $1 / 1(100 \%)$ \\
\hline Group 2 & & $9(27 \%)$ & & & $7 / 188(4 \%)^{*}$ \\
\hline t021 & 15-12----16-02-16-----------02-25-17-24--------- & $4(12 \%)$ & $\operatorname{del} \mathrm{D}(1)$ & $\operatorname{ins} C 2(3)$ & $3 / 57(5 \%)$ \\
\hline t298 & 15-12----16-02----------------------17-24---------- & $1(3 \%)$ & & delG-insB (1) & $1 / 5(20 \%)$ \\
\hline t10173 & 15-12-02-16-02----25-17-25-02-25-17-24-24------- & $1(3 \%)$ & & $\operatorname{insC2}(1)$ & $1 / 1(100 \%)$ \\
\hline t012 & 15-12----16-02-16----------02-25-17-24-24------ & $2(6 \%)$ & & $\begin{array}{l}\text { delE (1); } \\
\text { insC2 (1) }\end{array}$ & $2 / 123(2 \%)$ \\
\hline t6803 & 15-12----16-02-16-----------02-25-17-24-24-17-24 & $1(3 \%)$ & delD-insA (1) & & $0 / 2(0 \%)$ \\
\hline Group 3 & & $3(9 \%)$ & & & - \\
\hline t084 & $07-23-12-34-34-12-12-23-02-12-23$ & $1(3 \%)$ & delH (1) & & - \\
\hline t085 & $07-23-12-34-34-12----23-02-12-23$ & $2(6 \%)$ & delD (1); delA (1) & & - \\
\hline Group 4 & & $4(12 \%)$ & & & $3 / 74(4 \%)^{*}$ \\
\hline t280 & 04------------20-17-12-12--17------------- & $1(3 \%)$ & & delG-insB (1) & $1 / 4(25 \%)$ \\
\hline t227 & 04----------------12-12--17------------ & $1(3 \%)$ & delD (1) & & $0 / 3(0 \%)$ \\
\hline t078 & $04-21^{\mathrm{a}}-12^{\mathrm{b}}-41^{\mathrm{c}}-20-17-12-12-17--$ & $1(3 \%)$ & & delE (1) & $1 / 26(4 \%)$ \\
\hline $\mathrm{t} 216$ & 04------20-17--20-17-------------31 d $-16^{\mathrm{e}}-34^{\mathrm{f}}$ & $1(3 \%)$ & & delG-insB (1) & $1 / 41(2 \%)$ \\
\hline Group 5 & & $3(9 \%)$ & & & $1 / 92(1 \%)^{*}$ \\
\hline t032 & $26-23-23-13-23-31-29-17-31-29-17-25-17-25-16-28$ & $2(6 \%)$ & delD (1) & delE (1) & $1 / 79(1 \%)$ \\
\hline t223 & 26-23----13-23------------059-17-25-17-25-16-28 & $1(3 \%)$ & delD (1) & & $0 / 13(0 \%)$ \\
\hline Singletons & & $7(21 \%)$ & & & - \\
\hline t213 & $07-23-12-21-24-33-22-17$ & $3(9 \%)$ & delD (3) & & - \\
\hline t6792 & $08-16-02-16-17-13-17-13-17-16-34$ & $1(3 \%)$ & delD (1) & & - \\
\hline t6417 & $14-44-13-12-17-13-12-17-17-17-23-18$ & $1(3 \%)$ & dell (1) & & - \\
\hline t530 & $11-19-12-21-17-34-24-34-16$ & $1(3 \%)$ & & delE (1) & $1 / 3(33 \%)^{*}$ \\
\hline t7960 & $299-25-17-17-16-16-16-16$ & $1(3 \%)$ & dell-insC1 (1) & & - \\
\hline Total & & $33(100 \%)$ & & & \\
\hline
\end{tabular}

* $\mathrm{P}<0.0001$ comparing four groups of spa-types and the singleton with rearrangements affecting spa-typing $(5 \times 2$ Fisher's exact test).

$21^{\mathrm{a}}$ repeat differing in one base from repeats 12 and 20

$12^{\mathrm{b}}$ repeat differing in two bases from repeats 20 .

$41^{\mathrm{c}}$ repeat differing in one base from repeat 17.

$31^{\mathrm{d}}$ repeat differing in two bases from repeat 16 .

$16^{\mathrm{e}}$ repeat differing in one base from repeat 17.

$34^{f}$ repeat differing in two bases from repeats 12 and 20

$05^{9}$ repeat differing in one base from repeat 29.

affected by these rearrangements, a significantly higher proportion compared with the $1-4 \%$ in other groups or the singleton $\mathrm{t} 530$ ( $\mathrm{p}<0.0001)$. Therefore, spa-type $\mathrm{t} 571$ and its closely related variants such as t3085 may well be underrepresented in most $S$. aureus studies based on spa-typing, as they could not be typed with the standard set of primers when common deletions are present.

Interestingly, spa-type t571 belongs to clonal lineage ST398 that contains MRSA and MSSA strains common among livestock. Spa-type t571 is closely related to type t011 and t034, all most commonly associated with pigs [36-40]. These spa-types have been found less commonly in dogs, cats and horses, and occasionally in cattle and poultry $[41,42]$. Large-scale screening of pigs [36] showed that $60 \%$ of them carried t034, $14 \%$ t 1255 and $1.5 \%$ t571. Although ST398-associated spa-types have been rarely found among the general human population, they have been found more commonly in farmers 
working with pigs $[36,37]$. Veterinary personnel and pet owners are also more likely to carry these animal-related types [43]. Recent studies have also reported the emergence of livestock-associated MRSA clones of S. aureus ST398 causing bacteraemias in humans, supporting animal-independent transmission of such strains between humans $[44,45]$.

It is unclear why ST398 S. aureus strains commonly found in livestock frequently develop deletions in the IgG-binding part of protein A gene after transmission to humans. One possible explanation is that this might be a part of $S$. aureus strain adaptation to a different immune background where protein A plays a major role $[7,8,12]$. Another explanation might be that the livestock associated strains have more rearrangements in the spa-gene prior the transmission to humans due to high level of antibiotic exposure in food-animal production [46-48]. Nevertheless, our findings highlight the potential for these strains to have been substantially under-represented in epidemiological studies to date, and for strains formerly nottypeable using standard methods to be a source of bias.

\section{Conclusions}

Spa-typing is a common technique used to classify $S$. aureus strains in clinical practice (eg outbreak management) and research. Rearrangements in the IgG-binding region of the spa-gene make strains "non-typeable" with commonly used primers. Using a novel primer, we typed $100 \%$ of samples and identified eight novel spa-gene variants, plus one previously described; three of these rearrangements cause strains to be designated as "nontypeable" using current spa-typing methods.

Spa-typing of 6110 S. aureus isolates showed that 1.8\% of samples from $1.8 \%$ community carriers and $0.6 \%$ of samples from $0.7 \%$ inpatients were formerly non-typeable. We also found evidence of mixed colonization with strains with and without gene rearrangements, and estimated that up to $13 \%$ of carriers are colonized with "hidden" S. aureus with deletions/insertions in the IgG-binding region at some point. Using standard primers therefore underestimates spa-type diversity. We also found evidence of inpatients acquiring spa-gene deletions de novo during a hospital admission, suggesting that antibiotic pressure might be one factor driving genetic rearrangements in the $S$. aureus protein A gene. Finally, we found that deletions formerly causing strains to be designated as "non-typeable" were over-represented in clonal lineages related to livestock, indicating that these may well be have been underrepresented in most $S$. aureus studies. This new improved spa-typing protocol therefore enables previously overlooked $S$. aureus strains to be typed and therefore contribute to our understanding of diversity, carriage and transmission of $S$. aureus strains in community and hospitals.

\section{Additional files}

Additional file 1: Table S1. Swab data for individuals with

rearrangements in the spa-gene.

Additional file 2: Table S2. Association between rearrangements in the spa-gene and spa-types.

\section{Competing interests}

All authors declare that they have no competing interests.

\section{Authors' contributions}

AAV carried out laboratory experiments, participated in the analysis of data and writing of the manuscript, RF contributed to the collection and processing of samples for the study, RRM contributed to the design of the sample collection and sample database development, KK supervised recruitment of participants, as well as collection and processing of samples for the study, HG recruited participants for the study, DHW contributed in the design of the study and laboratory experiments, RB participated in the design of the study, DWC participated in the design of the study and contributed in the drafting of the manuscript, ASW performed statistical analysis and participated in the writing of the manuscript. All authors read and approved the final manuscript.

\section{Acknowledgments}

The authors wish to thank Dr. Teresa Street for discussion of the laboratory results, Dr. Kate Dingle for the comments on the manuscript, Ms. Alison Vaughan and Mr. David Griffiths for their assistance in the laboratory. This study was supported by the Oxford NIHR Biomedical Research Centre and the UKCRC Modernising Medical Microbiology Consortium, with the latter funded under the UKCRC Translational Infection Research Initiative supported by Medical Research Council, Biotechnology and Biological Sciences Research Council and the National Institute for Health Research on behalf of the Department of Health (Grant G0800778) and The Wellcome Trust (Grant 087646/Z/08/Z).

\section{Author details}

${ }^{1}$ Nuffield Department of Clinical Medicine, University of Oxford, John Radcliffe Hospital, Level 7, Room 7724, Oxford OX3 9DU, United Kingdom. ${ }^{2}$ National Institute for Health Research (NIHR) Oxford Biomedical Research Centre, John Radcliffe Hospital, Oxford OX3 9DU, United Kingdom. ${ }^{3}$ Department of Molecular Microbiology, John Innes Centre, Norwich Research Park, Norwich NR4 7UH, United Kingdom. ${ }^{4}$ School of Population and Public Health, University of British Columbia, British Columbia Centre for Disease Control, 655 West 12th Avenue, Vancouver, BC V5Z 4R4, Canada. ${ }^{5}$ The Jenner Institute, University of Oxford, Roosevelt Drive, Oxford OX3 7DQ, United Kingdom. ${ }^{6}$ Wellcome Trust Centre for Human Genetics, University of Oxford, Roosevelt Drive, Oxford OX3 7BN, United Kingdom.

${ }^{7}$ Gasteroenterology Research Office, Great Ormond Street Hospital for Children NHS Foundation Trust, Great Ormond Street, London WC1N 3JH, United Kingdom. ${ }^{8}$ Nuffield Department of Primary Care Health Sciences, University of Oxford, Oxford OX1 2ET, United Kingdom.

Received: 11 December 2013 Accepted: 4 March 2014 Published: 12 March 2014

\section{References}

1. Eriksen NH, Espersen F, Rosdahl VT, Jensen K: Carriage of Staphylococcus aureus among 104 healthy persons during a 19-month period. Epidemiol Infect 1995, 115(1):51-60.

2. Kluytmans J, van Belkum A, Verbrugh H: Nasal carriage of Staphylococcus aureus: epidemiology, underlying mechanisms, and associated risks. Clin Microbiol Rev 1997, 10(3):505-520.

3. Skramm I, Moen AE, Bukholm G: Nasal carriage of Staphylococcus aureus: frequency and molecular diversity in a randomly sampled Norwegian community population. APMIS 2011, 119(8):522-528.

4. Cole AM, Tahk S, Oren A, Yoshioka D, Kim YH, Park A, Ganz T: Determinants of Staphylococcus aureus nasal carriage. Clin Diagn Lab Immunol 2001, 8(6):1064-1069. 
5. Choi CS, Yin CS, Bakar AA, Sakewi Z, Naing NN, Jamal F, Othman N: Nasal carriage of Staphylococcus aureus among healthy adults. J Microbiol Immunol Infect 2006, 39(6):458-464.

6. Mahmutovic Vranic S, Puskar M: Staphylococcus aureus carriage among medical students. Med Glas Ljek komore Zenicko-doboj kantona 2012, 9(2):325-329.

7. Foster TJ: Immune evasion by staphylococci. Nat Rev Microbiol 2005, 3(12):948-958

8. Foster TJ, McDevitt D: Surface-associated proteins of Staphylococcus aureus: their possible roles in virulence. FEMS Microbiol Lett 1994, 118(3):199-205.

9. Guss B, Uhlen M, Nilsson B, Lindberg M, Sjoquist J, Sjodahl J: Region X, the cell-wall-attachment part of staphylococcal protein A. Eur J Biochem 1984, 138(2):413-420.

10. Uhlen $M$, Lindberg $M$, Philipson $L$ : The gene for staphylococcal protein A. Immunol Today 1984, 5(8):244-248.

11. Uhlen M, Guss B, Nilsson B, Gatenbeck S, Philipson L, Lindberg M: Complete sequence of the staphylococcal gene encoding protein $A$. A gene evolved through multiple duplications. J Biol Chem 1984 , 259(3):1695-1702.

12. Fournier B, Philpott DJ: Recognition of Staphylococcus aureus by the innate immune system. Clin Microbiol Rev 2005, 18(3):521-540.

13. Strommenger B, Braulke C, Heuck D, Schmidt C, Pasemann B, Nubel U, Witte W: spa Typing of Staphylococcus aureus as a Frontline Tool in Epidemiological Typing. J Clin Microbio/ 2008, 46(2):574-581.

14. Baum C, Haslinger-Loffler B, Westh H, Boye K, Peters G, Neumann C, Kahl BC: Non-spa-typeable clinical Staphylococcus aureus strains are naturally occurring protein A mutants. J Clin Microbiol 2009, 47(11):3624-3629.

15. Palmqvist N, Foster T, Tarkowski A, Josefsson E: Protein A is a virulence factor in Staphylococcus aureus arthritis and septic death. Microb Pathog 2002, 33(5):239-249.

16. Patel AH, Kornblum J, Kreiswirth B, Novick R, Foster TJ: Regulation of the protein A-encoding gene in Staphylococcus aureus. Gene 1992, 114(1):25-34.

17. Patel AH, Nowlan P, Weavers ED, Foster T: Virulence of protein A-deficient and alpha-toxin-deficient mutants of Staphylococcus aureus isolated by allele replacement. Infect Immun 1987, 55(12):3103-3110.

18. Poston SM, Glancey GR, Wyatt JE, Hogan T, Foster TJ: Co-elimination of mec and spa genes in Staphylococcus aureus and the effect of agr and protein A production on bacterial adherence to cell monolayers. J Med Microbiol 1993, 39(6):422-428

19. Stutz K, Stephan R, Tasara T: SpA, ClfA, and FnbA genetic variations lead to Staphaurex test-negative phenotypes in bovine mastitis Staphylococcus aureus isolates. J Clin Microbiol 2011, 49(2):638-646.

20. Kahl BC, Mellmann A, Deiwick S, Peters G, Harmsen D: Variation of the polymorphic region $\mathrm{X}$ of the protein $\mathrm{A}$ gene during persistent airway infection of cystic fibrosis patients reflects two independent mechanisms of genetic change in Staphylococcus aureus. J Clin Microbiol 2005, 43(1):502-505.

21. Finck-Barbancon $V$, Prevost G, Mazurier I, Piemont Y: A structurally novel staphylococcal protein A from the V8 strain. FEMS Microbiol Lett 1992, 70 (1):1-8.

22. Guss B, Leander K, Hellman U, Uhlen M, Sjoquist J, Lindberg M: Analysis of protein $\mathrm{A}$ encoded by a mutated gene of Staphylococcus aureus Cowan I. Eur J Biochem 1985, 153(3):579-585.

23. Movitz J, Masuda S, Sjoquist J: Physico- and immunochemical properties of staphylococcal protein A extracellularly produced by a set of mutants from Staphylococcus aureus Cowan I. Microbiol Immunol 1979, 23(2):51-60.

24. Lindmark R, Movitz J, Sjoquist J: Extracellular protein A from a methicillinresistant strain of Staphylococcus aureus. Eur J Biochem 1977, 74(3):623-628.

25. Miller R, Walker AS, Godwin H, Fung R, Votintseva A, Bowden R, Mant D, Peto TE, Crook DW, Knox K: Dynamics of acquisition and loss of carriage of Staphylococcus aureus strains in the community: The effect of clonal complex. J Infect 2014. doi:10.1016/j.jinf.2013.12.013.

26. Williamson SR, Walker AS, Knox KA, Votintseva A, Fung RKY, O'Connor L, Godwin H, Finney JM, Pill G, Moroney R, O'Sullivan OR, Oakley S, Peto TEA, Crook D, on behalf of the Infections in Oxfordshire Research Database (IORD): Comparison of Staphylococcus aureus acquisition and transmission rates in 3 wards using spa typing. In IDweek 2012, October
16-21. San Diego: The Infectious Disease Society of America (IDSA): 2012. Abstract 401.

27. Votintseva AA, Miller RR, Fung R, Knox K, Godwin H, Peto TE, Crook DW, Bowden R, Walker AS: Multiple-strain colonization in nasal carriers of Staphylococcus aureus. J Clin Microbiol 2014. doi:10.1128/JCM.03254-13.

28. Shopsin B, Gomez M, Montgomery SO, Smith DH, Waddington M, Dodge DE, Bost DA, Riehman M, Naidich S, Kreiswirth BN: Evaluation of protein A gene polymorphic region DNA sequencing for typing of Staphylococcus aureus strains. J Clin Microbiol 1999, 37(11):3556-3563.

29. Harmsen D, Claus H, Witte W, Rothganger J, Turnwald D, Vogel U: Typing of methicillin-resistant Staphylococcus aureus in a university hospital setting by using novel software for spa repeat determination and database management. J Clin Microbio/ 2003, 41(12):5442-5448.

30. Mellmann A, Weniger T, Berssenbrugge C, Rothganger J, Sammeth M, Stoye J, Harmsen D: Based Upon Repeat Pattern (BURP): an algorithm to characterize the long-term evolution of Staphylococcus aureus populations based on spa polymorphisms. BMC Microbio/ 2007, 7:98.

31. Bzymek M, Lovett ST: Instability of repetitive DNA sequences: the role of replication in multiple mechanisms. Proc Natl Acad Sci U S A 2001, 98(15):8319-8325.

32. Bzymek M, Lovett ST: Evidence for two mechanisms of palindromestimulated deletion in Escherichia coli: single-strand annealing and replication slipped mispairing. Genetics 2001, 158(2):527-540.

33. Bzymek M, Saveson CJ, Feschenko W, Lovett ST: Slipped misalignment mechanisms of deletion formation: in vivo susceptibility to nucleases. J Bacteriol 1999, 181(2):477-482.

34. Lopez E, Elez M, Matic I, Blazquez J: Antibiotic-mediated recombination: ciprofloxacin stimulates SOS-independent recombination of divergent sequences in Escherichia coli. Mol Microbiol 2007, 64(1):83-93.

35. Young BC, Golubchik T, Batty EM, Fung R, Larner-Svensson H, Votintseva AA, Miller RR, Godwin H, Knox K, Everitt RG, Igbal Z, Rimmer AJ, Cule M, Ip CL, Didelot X, Harding RM, Donnelly P, Peto TE, Crook DW, Bowden R, Wilson DJ: Evolutionary dynamics of Staphylococcus aureus during progression from carriage to disease. Proc Natl Acad Sci U S A 2012, 109 (12):4550-4555.

36. Khanna T, Friendship R, Dewey C, Weese JS: Methicillin resistant Staphylococcus aureus colonization in pigs and pig farmers. Vet Microbiol 2008, 128(3-4):298-303.

37. Weese JS: Methicillin-resistant Staphylococcus aureus in animals. ILAR J 2010, 51(3):233-244.

38. Oppliger A, Moreillon P, Charriere N, Giddey M, Morisset D, Sakwinska O: Antimicrobial resistance of Staphylococcus aureus acquired by pig farmers from pigs. Appl Environ Microbiol 2012, 78(22):8010-8014.

39. Osadebe LU, Hanson B, Smith TC, Heimer R: Prevalence and Characteristics of Staphylococcus aureus in Connecticut Swine and Swine Farmers. Zoonoses Public Health 2012, 60(3):234-43.

40. Verhegghe $M$, Pletinckx LJ, Crombe F, Vandersmissen T, Haesebrouck F, Butaye P, Heyndrickx M, Rasschaert G: Methicillin-Resistant Staphylococcus aureus (MRSA) ST398 in Pig Farms and Multispecies Farms. Zoonoses Public Health 2012, 60(5):366-374.

41. Hasman H, Moodley A, Guardabassi L, Stegger M, Skov RL, Aarestrup FM: Spa type distribution in Staphylococcus aureus originating from pigs, cattle and poultry. Vet Microbiol 2010, 141(3-4):326-331.

42. Witte W, Strommenger B, Stanek C, Cuny C: Methicillin-resistant Staphylococcus aureus ST398 in humans and animals, Central Europe. Emerg Infect Dis 2007, 13(2):255-258.

43. Moodley A, Stegger M, Bagcigil AF, Baptiste KE, Loeffler A, Lloyd DH, Williams NJ, Leonard N, Abbott Y, Skov R, Guardabassi L: spa typing of methicillin-resistant Staphylococcus aureus isolated from domestic animals and veterinary staff in the UK and Ireland. $J$ Antimicrob Chemother 2006, 58(6):1118-1123

44. Porphyre T, Giotis ES, Lloyd DH, Stark KD: A metapopulation model to assess the capacity of spread of meticillin-resistant staphylococcus aureus ST398 in humans. PLoS One 2012, 7(10):e47504

45. Verkade E, Bergmans AM, Budding AE, van Belkum A, Savelkoul P, Buiting $A G$, Kluytmans J: Recent emergence of staphylococcus aureus clonal complex 398 in human blood cultures. PLoS One 2012, 7(10):e41855.

46. Clark S, Daly R, Jordan E, Lee J, Mathew A, Ebner P: Extension education symposium: the future of biosecurity and antimicrobial use in livestock production in the United States and the role of extension. J Anim Sci 2012, 90(8):2861-2872 
47. Sapkota AR, Lefferts LY, McKenzie S, Walker P: What do we feed to food-production animals? A review of animal feed ingredients and their potential impacts on human health. Environ Health Perspect 2007, 115(5):663-670.

48. Zhou LJ, Ying GG, Liu S, Zhang RQ, Lai HJ, Chen ZF, Pan CG: Excretion masses and environmental occurrence of antibiotics in typical swine and dairy cattle farms in China. Sci Total Environ 2012, 444C:183-195.

doi:10.1186/1471-2180-14-63

Cite this article as: Votintseva et al.: Prevalence of Staphylococcus aureus protein A (spa) mutants in the community and hospitals in Oxfordshire. BMC Microbiology 2014 14:63.

\section{Submit your next manuscript to BioMed Central and take full advantage of:}

- Convenient online submission

- Thorough peer review

- No space constraints or color figure charges

- Immediate publication on acceptance

- Inclusion in PubMed, CAS, Scopus and Google Scholar

- Research which is freely available for redistribution 